\title{
Stability analysis of a novel four-wheeled motorcycle in straight running
}

\author{
Francesco Bucchi, Francesco Cerù and \\ Francesco Frendo
}

Received: date / Accepted: date \begin{abstract}
the scooter to roll freely as a standard motorcycle. straight without any control system.

F. Bucchi

Department of Civil and Industrial Engineering

Università di Pisa

Largo Lucio Lazzarino, 56122, Pisa

Tel.: +39-050-2218019

E-mail: francesco.bucchi@unipi.it

F. Cerù

Department of Civil and Industrial Engineering

Università di Pisa

Largo Lucio Lazzarino, 56122, Pisa

F. Frendo

Department of Civil and Industrial Engineering

Università di Pisa

Largo Lucio Lazzarino, 56122, Pisa

Tel.: +39-050-2218074

E-mail: frendo@ing.unipi.it
\end{abstract}

Abstract This paper deals with the stability analysis of a novel four wheeled tilting motorcycle. This new kind of vehicle is equipped with two front steering wheels and two rear wheels; the front and rear suspension mechanisms allow

An analytical model of the vehicle with locked suspensions, rigid and lenticular tires was developed in order to study the stability of the system in straight running and to carry out a modal analysis focusing on the main normal modes. The frame and all the vehicle components were considered as rigid bodies, the driver was considered fixed to the frame and the tires behavior was described by a linear function of the roll and side-slip angles. The action of the driver on the steering was not taken into account, considering the vehicle running in

The stability analysis shows that the eigenmodes of the four wheeler have several common features with the capsize, weave, wobble and rear wobble modes which characterize the dynamics of two and three wheeled motorcy- 
cles. A sensitivity analysis showing the influence of the front trail on stability and the vehicle eigenmodes is presented. A graphical and intuitive representation of the eigevenctors is also given.

Keywords Vehicle dynamics, motorcycle, 4-wheeler, tilting vehicle, stability analysis, capsize, weave, wobble.

\section{Introduction}

This study deals with the stability analysis of a novel four-wheeled tilting vehicle equipped with two front steering wheels and two rear wheels. This new kind of vehicle was designed with the aim of improving passive safety and conceived to develop a new kind of urban mobility, suitable also for people that usually drive cars and do not consider two-wheelers as a safe and comfortable alternative. Indeed, this new vehicle can be regarded as a further development of the already available on the market three wheeled motorcycles.

Nowadays, the need of developing new forms of urban mobility is of great concern; it is common experience that traffic is chaotic and, frequently, pollution caused by vehicles gets over the critical levels in many cities and for this reason the European Commission (EC) is promoting research in this field. The development of a new type of vehicle endowed with an environmentally low-impact propulsion system and with a novel layout represents an interesting answer to this need.

The Department of Civil and Industrial Engineering of the University of Pisa is involved in the RESOLVE research project (Range of Electric Solution for L-category Vehicles) funded by the EC in the Horizon 2020 framework programme. The aim is to develop two prototypes of novel four-wheeled tilting vehicles (Fig. 1) belonging to the L-category vehicles, L2e and L6e category, respectively, [EU Regulation(2013)]. These ELVs (Electric L-category Vehicles) will be characterized by low level of energy consumption, high safety requirements and low cost. To achieve that, they will be equipped with modular and scalable electric powertrains and battery architectures.

Since the layout of those vehicles is completely new, an in depth analysis of the dynamic properties was mandatory, starting from the stability analysis.

The study of the stability of motorcycles is a common subject in the literature. The first stability analyses were carried out by [Sharp(1971)] who considered the vehicle composed by two rigid bodies, i.e. the front system and the main frame, joined by a revolute joint at the steering head. Three main modes were identified as a function of the longitudinal speed, namely the capsize, the weave and the wobble. Sharp also studied the principal modal shapes when the vehicle was in a corner [Sharp(2001)] and developed more accurate models including a precise behavior of tires [Sharp et al.(2004)].

Subsequently, Sharp improved his model in order to include the suspensions deformation, more accurate tire characteristics and tire radial stiffness [Sharp and Limebeer(2001)]. In [Limebeer et al.(2001)] the authors also investigated the effects of acceleration and deceleration on motorcycle stabil- 


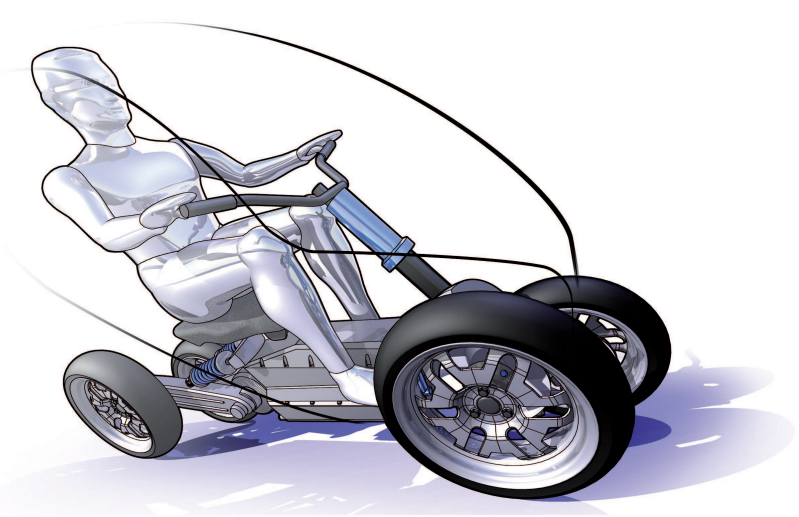

Fig. 1: RESOLVE 4-wheeler tilting vehicle concept.

ity. Other mathematical models of motorcycle have been studied in the last years; in [Frendo(2006)] a motorcycle model was presented, in which the influence of the tire model, of the gyroscopic effects of wheels and engine and of the front vehicle geometry on the handling parameters was studied. In [Nishimi et al.(1985)] a model where the rider has two degrees of freedom with respect to the main frame was developed: the lower body of the driver can shift laterally, while the upper is free to roll. In [Limebeer et al.(2001)] the stability was also studied under acceleration and braking, while in [Evangelou et al. (2008)] the effect of non-flat road is considered. In [Cossalter et al.(2004)] a modal analysis of a sport motorcycle was presented; the tires were considered having their real tread geometry and radial stiffness, and the position of the tire-road contact point was considered as function of the camber angle and tire deformations.

Recently, a new kind of scooters was introduced on the market, which are equipped with two front wheels and a front suspension mechanism that allows the vehicle the freely roll and, as a consequence, to be perceived and driven as a standard motorcycle. The stability analysis of a three wheeler was presented in several papers [Sponziello et al.(2010)]. In [Sponziello et al.(2009), Bartaloni et al.(2007)] the stability analysis of a threewheeler based on the first Sharp model was presented. The scooter was made up of rigid bodies, the suspensions were locked and the wheels were considered rigid and lenticular. The wheel-road contact was represented by a point and the lateral tire forces were linear functions of side-slip and camber angles. The driver was rigidly joined to the main frame. The authors studied the stability of the vehicle in straight running and in turning. The results were compared with those of a two-wheeler with similar characteristics and showed how the weave mode for the three-wheeler was more stable. The wobble mode of the three-wheeler was very close to that of a two wheeler and the capsize was always unstable from zero to $60 \mathrm{~m} / \mathrm{s}$, while the two-wheeler had a stable capsize mode at low speed, that turned into an unstable mode over $8 \mathrm{~m} / \mathrm{s}$. 
Other papers deal with tilting three-wheeler, without analyzing the stability of the vehicle. In [Rajamani and Gohl(2003)] a mathematical model of a tilting three-wheeler is described and the effect of steering, tilting and gyroscopic moment is discussed on the basis of equations. In [Amati et al.(2011)] a mathematical model of a tilting three-wheeler is presented, which include both the simulation of dynamic maneuvers and the computation of eigenmodes. Concerning dynamic maneuvers, the trend of steering and roll versus time is compared, in order to validate the model. In [Sponziello et al.(2010)] the oscillations of the front mechanism of a tilting three-wheeler, equipped with traditional springs and dampers, are numerically evaluated and a mass damper is proposed in order to reduce possible vibrations excited by the road profile.

To the best of the authors' knowledge, currently no analysis can be found in the literature dealing with four-wheeler titling vehicles. For this reason, in this paper a mathematical model of a novel four-wheeler is discussed with reference to stability. This model was developed inspired to the two-wheeler Sharp model and to the-three wheeler model described by [Sponziello et al.(2009)]. The modal analysis of such a vehicle in straight running is presented in this paper. The natural modes of the vehicle were studied in order to establish any similarity with the modes of a two or three-wheeler. A sensitivity analysis to the front trail, which is the most influencing stability parameter, is also discussed.

A new graphical representation, inspired from the compass plot representation [Cossalter et al.(2011)], of the eigenmodes is also introduced to easily show, in a single plot, for each eigenmode, the shape of the eigenvector together with the related eigenvalue trend, as a function of the forward speed.

\section{Mathematical model of the vehicle}

\subsection{Description of the vehicle}

The vehicle, which is schematically shown in Fig. 2, is made up of the 23 bodies which can be split into three subsystems:

- the front tilting system (blue parts in Fig. 2);

- the main frame (black parts in Fig. 2);

- the rear tilting system (red parts in Fig. 2).

Both the front and rear systems are made up of 11 bodies, constrained in order to guarantee one degree of freedom (tilting) to each system. In particular, the front tilting mechanism is composed of two upper and two lower A-arms which are independently joined to the main frame by revolute joints. The left and right upper [lower] A-arms are independently connected to the frame by two co-axial revolute joints, which are schematically 


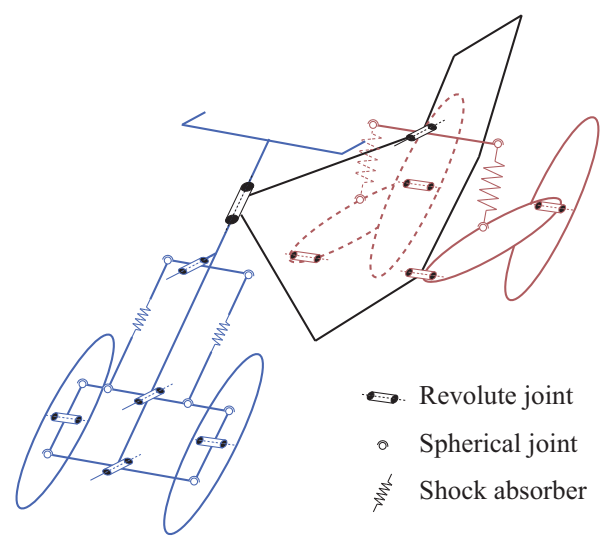

Fig. 2: Scheme of the 4-wheeler.

represented by a single revolute joint in Fig. 2. On both sides, a hubcarrier is joined to the horizontal arms by two spherical joints, which determine the steering axis of each front wheel. Each superior A-arm is connected to a central rocker arm by a shock absorber, guaranteeing the desired vertical stiffness and allowing the free roll of the vehicle. The steering system, which is not represented in the scheme, is composed of two steering rods which link the rotation of the handlebar to the steering motion of the wheels about the steering axes. The steering ratio between the the handlebar and the wheels is nearly unitary. The steering axis is defined by the line which connects the upper and lower spherical joints of the hub-carrier, and can be described by the parameters shown in Fig. 3.

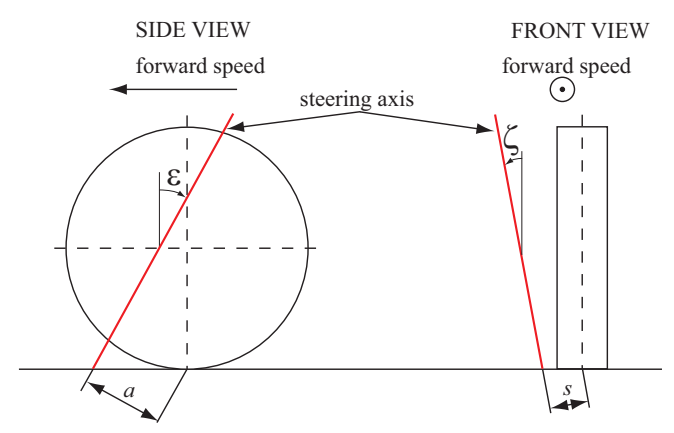

Fig. 3: Steering axis parameters: normal trail $(a)$, lateral trail $(s)$, caster angle $(\varepsilon)$, kingpin angle $(\zeta)$.

The rear tilting system is composed of two swingarms, one for each rear wheel, that are joined to the main frame by revolute joints. As for the front tilting mechanism, a shock absorber connects each swing arm with a central 


$\begin{array}{lcc}\text { Mass (including driver) } & (\mathrm{kg}) & 340 \\ \text { Wheelbase } & (\mathrm{m}) & 1.61 \\ \text { Front track } & (\mathrm{m}) & 0.70 \\ \text { Rear track } & (\mathrm{m}) & 0.44 \\ \text { CoG Height } & (\mathrm{m}) & 0.59 \\ \text { CoG Longitudinal distance from rear wheel } & (\mathrm{m}) & 0.86 \\ \text { Normal trail }(a) & (\mathrm{m}) & 0.04 \\ \text { Lateral trail }(s) & (\mathrm{m}) & 0.07 \\ \text { Caster angle }(\varepsilon) & (\mathrm{deg}) & 25 \\ \text { Kingpin angle }(\zeta) & (\mathrm{deg}) & 0\end{array}$

Table 1: Vehicle parameters

rocker arm, achieving the desired vertical stiffness and allowing free roll of the vehicle. The main frame, which is assumed as a unique rigid body, reproduces the mass and inertia of the frame itself, the powertrain elements, the petrol tank, the driver seat and the rider which is supposed to be rigidly fixed to it.

The front system is connected to the main frame by the revolute joint at the steering head and by a revolute joint at the front tilting bar. The rear system is constrained to the main frame by two co-axial revolute joints at the swing arms and by a revolute joint at the rear tilting bar.

\subsection{Mathematical model of the vehicle}

The following simplifying hypotheses were assumed to limit the complexity of the model:

- the model is made up of rigid bodies;

- the driver is fixed to the main frame and cannot move with respect to it;

- the position of the global center of gravity is assumed to be invariant in a vehicle reference frame;

- the road is flat and horizontal and the suspensions are assumed to be infinitely rigid;

- the kinematic pitch, due to the rotation of the front wheels along the steering axes, is neglected;

- the tires are assumed to be lenticular and rigid;

- the longitudinal tire slip is neglected.

It is worth noting that, due to the front and rear tilting mechanisms, the assumption of rigid suspensions does not allow the vehicle to bounce even if roll is possible.

The vehicle reference frame was assumed to have origin in $N$, which is the projection on the ground of the center of $\operatorname{mass} G$, made along the middleplane of the vehicle, and to have the z-axis always normal to the ground, the $\mathrm{x}$-axis along the vehicle longitudinal direction and directed forward and the $y$-axis consequently obtained according to a right-handed coordinate system (see Fig. 4). Point $N$ belongs to the vehicle roll axis, which, as in a standard motorcycle, can be obtained by the intersection 
between the ground plane and the middle plane of the vehicle, if the suspension are locked.

Basing on these hypotheses, the following state variables fully describe the model state (see also Fig. 4)

- longitudinal and lateral speeds ( $u$ and $v$ respectively) of the point $N$;

- yaw velocity of the main frame $r$;

- relative rotation between the front system and the rear frame along the steering head, namely the steering angle $\delta$;

- tilting of the main frame along the longitudinal axis $x$, namely the roll angle $\phi$ of the vehicle.

The initial position of the point $N$ was also assumed as the origin of the global reference frame.

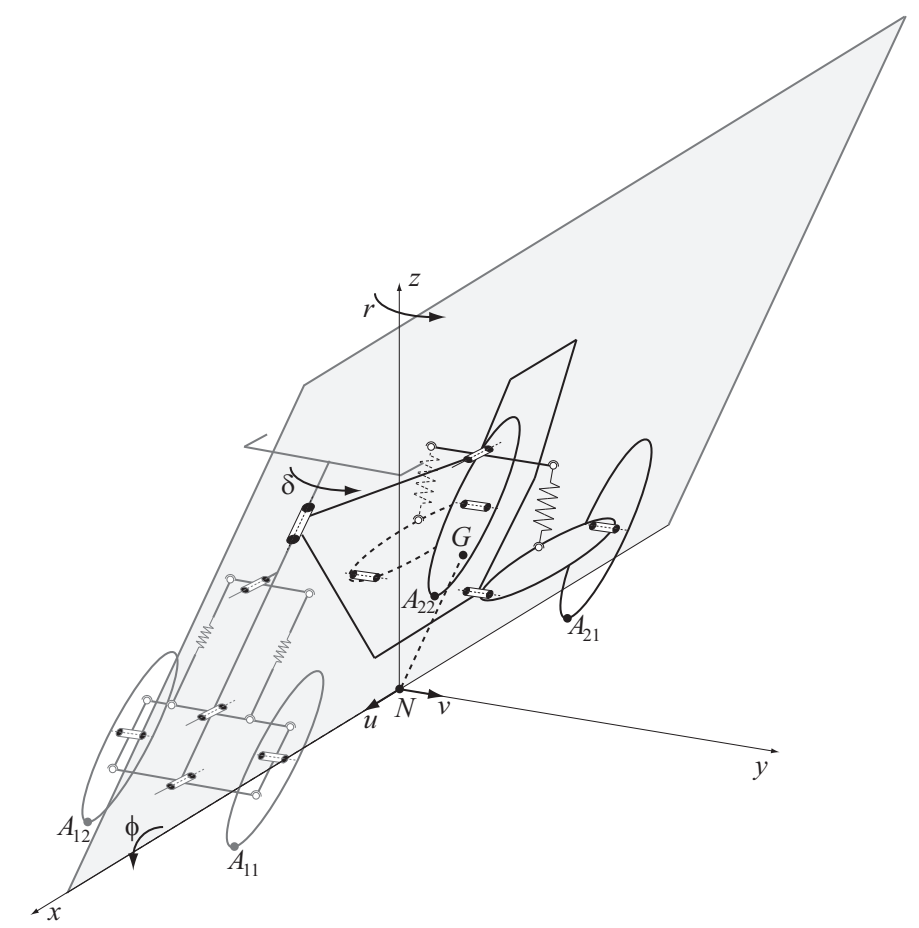

Fig. 4: State variables of the model. The position of point $N$ and contact points $A_{11}, A_{12}, A_{21}, A_{22}$ are also shown.

The angular velocity of the main frame system $\omega_{\mathrm{f}}$ is

$$
\omega_{\mathrm{f}}=r \mathbf{k}+\dot{\phi} \mathbf{i}
$$

and the velocity of the point $\mathrm{N}$ is

$$
\mathbf{V}_{\mathrm{N}}=u \mathbf{i}+v \mathbf{j} .
$$


Consequently, since $N$ lies on the roll axis and is a point belonging to the frame, the velocity of the center of mass $G$ is

$$
\mathbf{V}_{\mathrm{G}}=\mathbf{V}_{\mathrm{N}}+\omega_{\mathrm{f}} \times N G
$$

and its acceleration is

$$
\mathbf{a}_{\mathrm{G}}=\mathbf{a}_{\mathrm{N}}+\dot{\omega}_{\mathrm{f}} \times N G+\omega_{\mathrm{f}} \times\left(\omega_{\mathrm{f}} \times N G\right)
$$

where $\mathbf{a}_{\mathrm{N}}$ is the acceleration of the point $N$ and $\dot{\omega}_{\mathrm{f}}$ is the time-derivative of the angular velocity $\omega_{\mathrm{f}}$.

Starting from these values, the angular and translational velocity and acceleration of each body can be computed on the basis of kinematic constraints.

Equilibrium equations. The dynamic equations which allow the system to be simulated can be obtained following either the Newton-Euler or the Lagrange form. In the following the equations for the global equilibrium of the vehicle are derived by the Newton Eulero approach.

The global translation equilibrium can be expressed by the equation:

$$
\mathbf{F}_{11}+\mathbf{F}_{12}+\mathbf{F}_{21}+\mathbf{F}_{22}+m \mathbf{g}+\mathbf{F}_{\mathrm{a}}=m \mathbf{a}_{\mathrm{G}}
$$

where $\mathbf{F}_{\mathrm{ij}}$, with the first subscript $i$ identifying the axle $(i=1$ is front, $i=2$ is rear) and the second subscript $j$ identifying the side $(j=1$ is left, $j=2$ is right), are the resultant tire to road forces applied at the four wheels, $\mathbf{F}_{\mathrm{a}}$ is the aerodynamic drag force, $m$ is the total mass of the vehicle including the rider. Similarly, the rotational equilibrium equations about the vehicle center of gravity $G$ along the $x, y$ and $z$ axes is expressed by

$$
\begin{array}{r}
G A_{11} \times \mathbf{F}_{11}+G A_{12} \times \mathbf{F}_{12}+G A_{21} \times \mathbf{F}_{21}+G A_{22} \times \mathbf{F}_{22}+G C_{\mathrm{p}} \times \mathbf{F}_{\mathrm{a}}= \\
=\sum_{k}^{N}\left(G G^{k} \times m^{k} a_{\mathrm{G}^{k}}+\mathbf{I}_{\mathrm{G}^{k}} \dot{\omega}^{k}+\omega^{k} \times \mathbf{I}_{\mathrm{G}^{k}} \omega^{k}\right)
\end{array}
$$

where the left-hand side terms of the equation refer to the moment due to the external forces, while the right-hand side term is related to the moment due to the inertia forces, which is the time-derivative of the angular momentum. In particular, $G A_{\mathrm{ij}}$ are the the vectors connecting the center of mass $G$ to each wheel to road contact point $A_{\mathrm{ij}}, G C_{\mathrm{p}}$ is the vector connecting the center of mass to the aerodynamic drag force center of pressure, $G G^{k}$ is the vector connecting the vehicle center of mass to each part center of mass $G^{k}, \mathbf{I}_{\mathrm{G}^{k}}$ is the inertia tensor of each body about its own center of mass, $\omega^{k}$ and $\dot{\omega}^{k}$ are the angular velocity and the angular acceleration of each body respectively, both expressed in a body reference frame, and $N$ is the number of the bodies $(N=23$ in the present case).

Similarly, the equilibrium equations about the steering axis and the tilting lever axes (both front and rear) were obtained considering the external forces and intertia forces. 
Tires constitutive equations. For each wheel, the tire lateral force $F_{\text {yij }}$ was described by a linear differential equation for each lateral force $F_{\text {yij }}$

$$
F_{\text {yij }}=C_{\alpha} \alpha_{\text {yij }}+C_{\gamma} \gamma_{\text {yij }}
$$

where $C_{\alpha}$ and $C_{\gamma}$ are the tire slip stiffness and the camber stiffness respectively and $\alpha_{\text {yij }}$ ad $\gamma_{\text {yij }}$ are the slip angle and camber angle of each tire. The longitudinal components of rear tires forces $F_{\mathrm{x} 21}$ and $F_{\mathrm{x} 22}$ were assumed as inputs for the model and are defined subsequently.

Congruence equations. The set of equations was completed with the congruence equations, which define the slip and camber angles as function of the state variables $\delta$ and $\phi$ and the steering axis angles $\varepsilon$ and $\zeta$

$$
\begin{gathered}
\gamma_{11}=\gamma_{12}=\arccos \left[\cos (\phi)-2 \cos (\phi) \sin ^{2}\left(\frac{\delta}{2}\right)\left(\sin ^{2}(\varepsilon)+\cos ^{2}(\varepsilon) \sin ^{2}(\zeta)\right)+\right. \\
\left.+\sin (\phi)\left(\sin (\delta) \sin (\varepsilon)+\cos ^{2}(\varepsilon) \sin ^{2}\left(\frac{\delta}{2}\right) \sin (2 \zeta)\right)\right] \\
\gamma_{21}=\gamma_{22}=\phi
\end{gathered}
$$

The tire slip angles are obtained in the $x-y$ plane starting from the state variables $u, v$ and $r$ as usually done for cars single-track models [Guiggiani (2014)]

$$
\begin{gathered}
\alpha_{11} \simeq \alpha_{12} \simeq-\delta-\frac{v+r l}{u} \\
\alpha_{21} \simeq \alpha_{22} \simeq-\frac{v-r l}{u}
\end{gathered}
$$

were $l$ is the vehicle wheelbase.

Constant speed control. In this paper a straight line maneuver with free handlebar is considered. For this reason a control logic was defined only to keep the speed constant and no torque control was applied to the handlebar. A reference speed $u_{\mathrm{d}}$ was defined for each maneuver and the actual longitudinal speed $u$ was measured during the simulation. A proportional controller was set-up to determine the rear traction forces $F_{\mathrm{x} 21}$ and $F_{\mathrm{x} 22}$ as follows

$$
F_{\mathrm{x} 21}=F_{\mathrm{x} 22}=k\left(u_{\mathrm{d}}-u\right)
$$

where $k$ is the proportionality constant and it was determined by some preliminary simulations. 


\section{Stability analysis}

The mathematical model of the four-wheeler is ruled by 21 non-linear equations (9 equilibrium equations, 4 constitutive equations, 8 congruence equations) with 21 variables: longitudinal speed $u$, lateral speed $v$, yaw rate $r$, roll angle $\phi$, steering angle $\delta$, four vertical forces $F_{\mathrm{zij}}$, four lateral forces $F_{\text {yij }}$, four slip angles $\alpha_{\mathrm{ij}}$ and four camber angles $\gamma_{\mathrm{ij}}$.

After suitable substitutions the system was linearized and written in the state space form

$$
\dot{\mathbf{x}}=A(u) \mathbf{x}
$$

where $A(u)$ is the dynamic matrix and $\mathrm{x}$ is the vector of the state variables and $\dot{\mathbf{x}}$ is its time derivative. In this form the linear system is made up of 11 equations and the state vector $\mathbf{x}$ is made up of the following state variables

$$
\mathbf{x}=\left(F_{\mathrm{y} 11}, F_{\mathrm{y} 12}, F_{\mathrm{y} 21}, F_{\mathrm{y} 22}, u, v, \dot{\delta}, \dot{\phi}, r, \delta, \phi\right)
$$

The dynamic matrix of the system $A(u)$ is function of the forward speed $u$ and, consequently, the eigenvalues and eigenvectors are function of the speed as well.

In order to show the trend of the eigenvalues as a function of the forward speed, the range of speed between 0 and $60 \mathrm{~m} / \mathrm{s}$ was considered and it was discretized every $0.1 \mathrm{~m} / \mathrm{s}$. For each speed step the dynamic matrix $A(u)$ was computed and the eigenvalues and eigenvectors were obtained. It was necessary to implement a post-processing procedure in order to isolate the most interesting eigenvalues. Indeed, it is worth noting that the vector of the state variables is not homogeneous, the first four components are lateral forces, the following five are linear or rotational velocities and the last two are angles, which may be obtained by integration starting from the steering rate and the roll rate. For this reason, since the information related to the vehicle dynamics are all enclosed in the angular rate components $\dot{\delta}, \dot{\phi}$ and $r$, the study of the eigenmodes was reduced to these components of the eigenvectors. With reference to the Fig. 4, the roll rate is positive when the vehicle tilts on its right side, the steering rate is positive when the handlebar turns leftward and the yaw rate is positive when the main frame turns leftward (for an observer seated on the vehicle). Using these reduced eigenvectors it was possible to clearly identify all the modes of the four-wheeler.

Considering that an experimental validation was not possible, since no prototype exists, yet, an equivalent two-wheeler version of the developed model was obtained and validated by comparison with the benchmark model given in [Meijaard et al.(2007)] (results of this validation are omitted). 


\section{Results and discussion}

4.1 Eigenmodes analysis at different longitudinal speeds

The results of the analyses described in the previous section are shown Fig. 56 in terms of real and imaginary parts of the eigenvalues, respectively, as a function of the longitudinal speed $u$. The trend of the eigenvalues is similar to the trends already found for a two and a three-wheeler [Sponziello et al.(2009)]. Two eigenvalues are real along the whole investigated speed range, namely the capsize and the rear wobble. The weave mode is non-oscillatory below $5 \mathrm{~m} / \mathrm{s}$ and then becomes oscillatory with frequency below $2 \mathrm{~Hz}$. Finally the wobble is oscillatory above about $2 \mathrm{~m} / \mathrm{s}$ and its frequency is about $5 \mathrm{~Hz}$ for $u>5 \mathrm{~m} / \mathrm{s}$.

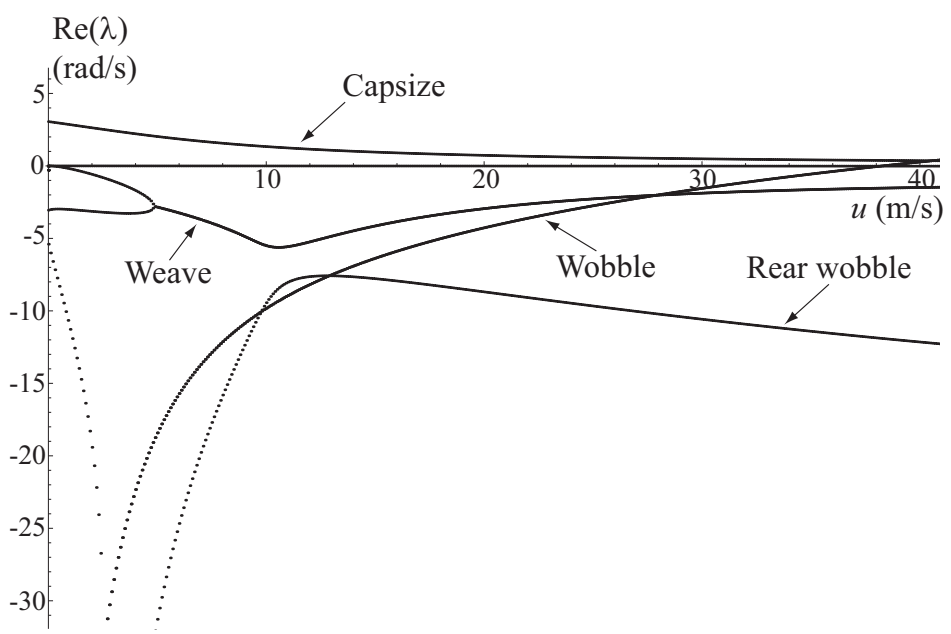

Fig. 5: Real part of the four-wheeler eigenvalues as a function of the longitudinal speed.

In order to investigate how the modal shapes are influenced by the longitudinal speed, an analysis of the eigenvectors was performed, considering the relevant components $r, \dot{\phi}$ and $\dot{\delta}$. The results of the analysis are shown in Fig. $7-10$ were the real part of each eigenvalue versus longitudinal speed is shown and, at four selected speed, i.e. $4 \mathrm{~m} / \mathrm{s}, 10 \mathrm{~m} / \mathrm{s}, 20 \mathrm{~m} / \mathrm{s}, 30 \mathrm{~m} / \mathrm{s}$, a map of the eigenvector components is shown. Each map is represented as a cartesian coordinate system having the real and the imaginary part of each eigenvector component on the horizontal and vertical axes, respectively. In order to give an idea of the relative magnitude of the eigenvectors components, two red circles were also drawn on each coordinate system, the smaller having unitary radius and the second having $5 \mathrm{rad} / \mathrm{s}$ radius. The eigenvector components were represented by a circle $(r)$, a diamond $(\dot{\phi})$ and a small cross $(\dot{\delta})$. If the eigenvector component amplitude exceeded $5 \mathrm{rad} / \mathrm{s}$, the eigenvector symbol was draft on 


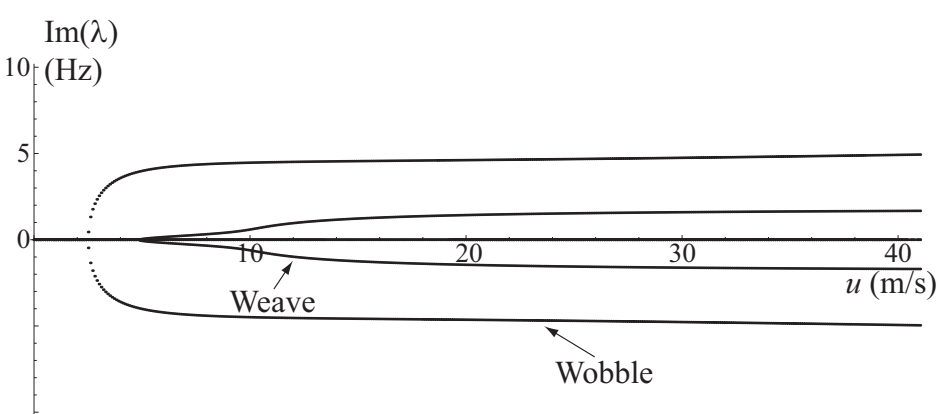

Fig. 6: Imaginary part of the four-wheeler eigenvalues as a function of the longitudinal speed.

the external circle and an outward arrow was added as in Fig. 8 and Fig. 9. The eigenvector components were calculated in order to have the amplitude of the yaw rate $r$ unitary and zero-phase.

Figure 7 shows the capsize mode which is non-oscillatory since its associate eigenvalues is always real; in the case of the analyzed four-wheeler vehicle the capsize eigenvalue is greater than zero, that means that the mode is unstable in the whole investigated speed range. Concerning the eigenvector, the roll rate $\dot{\phi}$ is always in counter-phase with respect to the yaw rate $r$; with reference to the vehicle scheme in Fig. 4, this means that if the vehicle yaw is leftward also the roll rate will be leftward. The $\dot{\phi}$ component is the largest at any speed value, even if its amplitude decreases as the longitudinal speed rises. The steering rate $\dot{\delta}$ is almost negligible expecially at high speed.

Figure 8 shows the weave mode, which is characterized by two different real eigenvalues at low longitudinal speed (non-oscillatory behavior) and two complex-conjugate eigenvalues (oscillatory behavior) at higher longitudinal speed; the real part of the eigenvalues is always negative. Concerning the eigenvectors, at $4 \mathrm{~m} / \mathrm{s}$, one eigenvector is mainly characterized by roll rate and steering rate, which are in counter phase; the other eigenvector has almost equivalent contributions of $r, \dot{\phi}$ and $\dot{\delta}$. At longitudinal speed above $5 \mathrm{~m} / \mathrm{s}$ the weave mode becomes oscillatory and its eigenvector is characterized by almost equivalents amounts of $r, \dot{\phi}$ and $\dot{\delta}$, with $\dot{\phi}$ and $\dot{\delta}$ in quasi-counter phase with respect to $r$. This means that when the yaw speed is leftward, also the roll speed is leftward while the steering speed is rightward, which is the typical behavior of the capsize mode. The wobble mode, represented in Fig. 9, is not influenced by speed in the examined range. The wobble eigenvalue is stable in almost all the considered longitudinal speed range and becomes unstable at about $38 \mathrm{~m} / \mathrm{s}$; this shall be taken in due consideration in case of vehicle operating at high speed. At very low speed, two different real eigenvalues were found which become two complex-conjugate eigenvalues at about $5 \mathrm{~m} / \mathrm{s}$. The main component of the wobble eigenvector is the steering rate which is in 


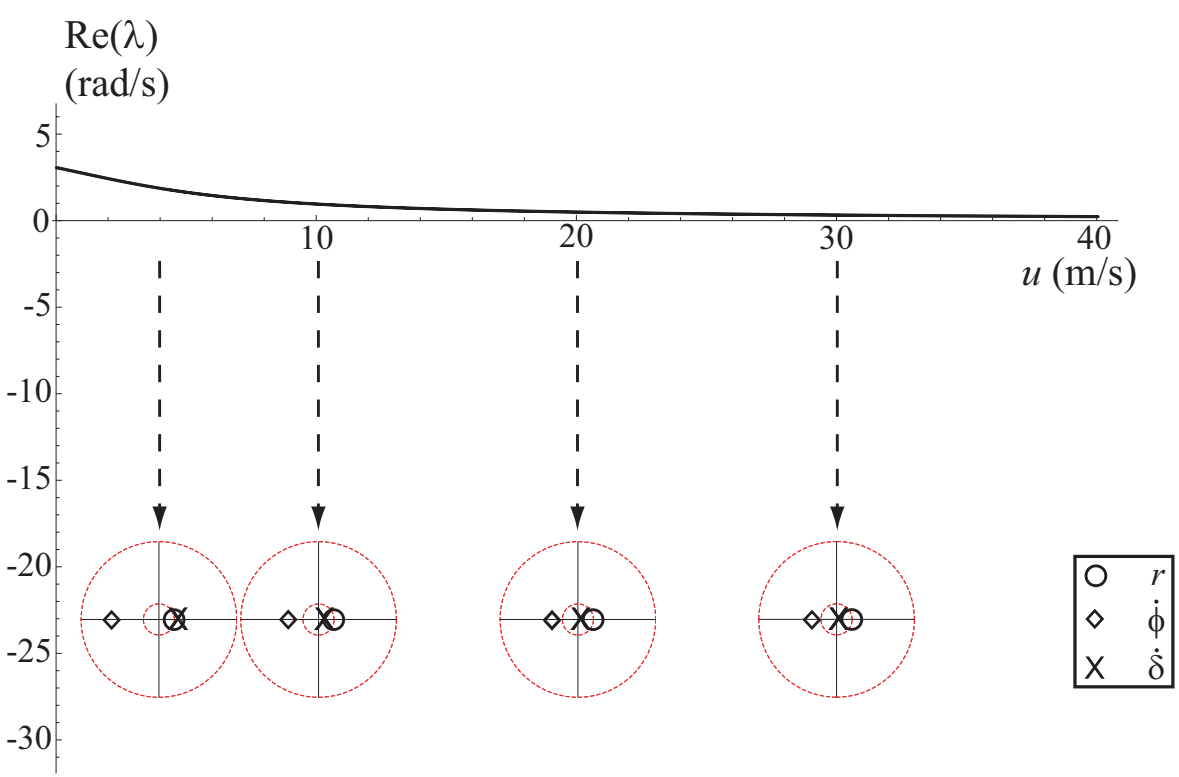

Fig. 7: Capsize eigenvalue and eigenvector representation versus longitudinal speed.

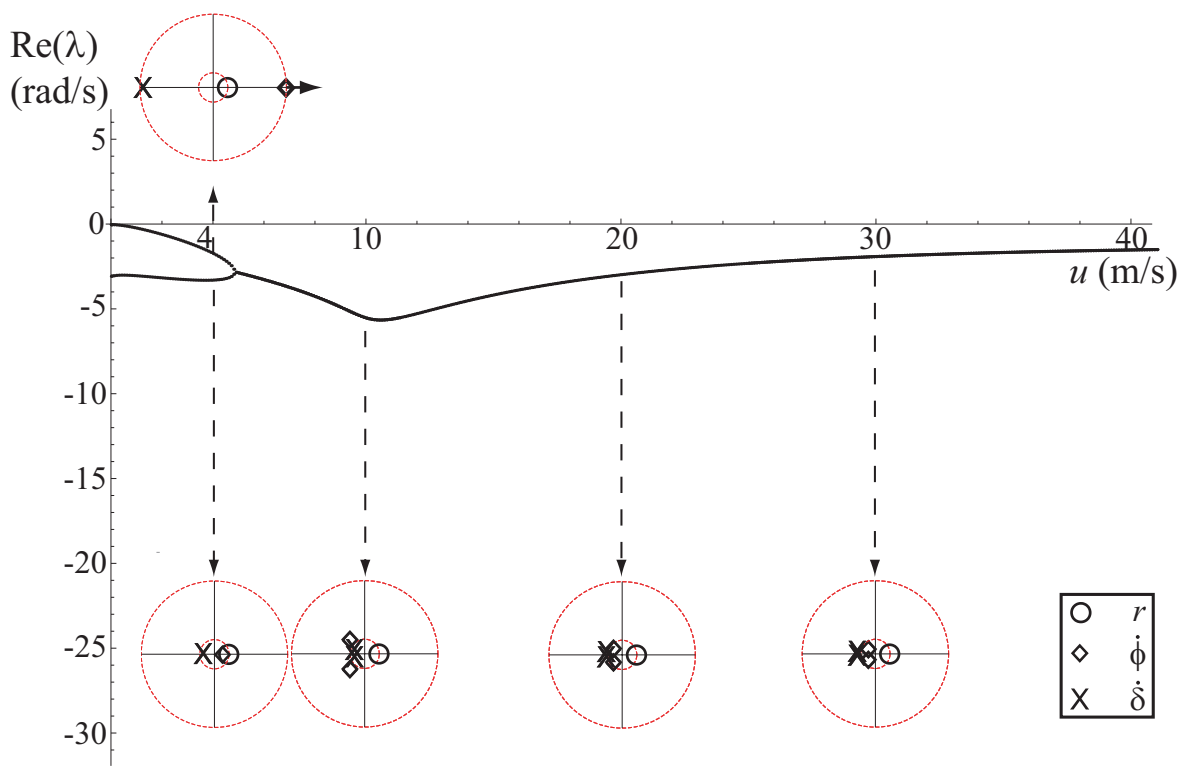

Fig. 8: Weave eigenvalue and eigenvector representation versus longitudinal speed. 


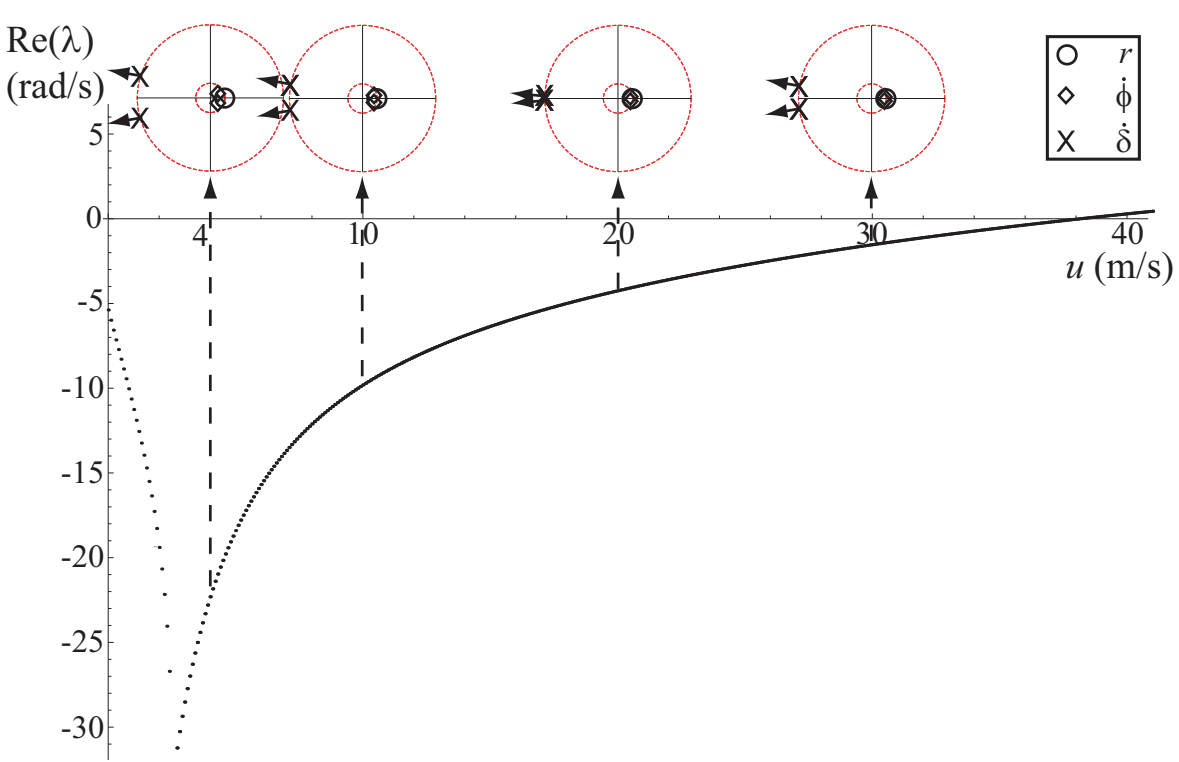

Fig. 9: Wobble eigenvalue and eigenvector representation versus longitudinal speed.

quasi-counter-phase with respect to yaw and roll rate in the whole investigated longitudinal speed range.

Finally, the rear wobble is shown in Fig. 10. This mode is non-oscillatory and it is strongly stable in the whole considered longitudinal speed range and for this reason it is usually not deeply investigated in the literature; in particular its eigenvector is not meaningful [Cossalter and Lot(2002)] and its yaw, roll rate and steering rate components significantly varies in the analyzed speed range.

\subsection{Sensitivity analysis}

Starting from the reference configuration of the four-wheeler considered in the previous section, the influence on the eigenvalues of the front trail $a$ was investigated. This parameter was selected since it was found as the most influencing parameter on stability. Three front trail values were investigated: a) the reference configuration, having front trail $a=0.04 \mathrm{~m}$, which gave the results presented in Fig. 5; b) a configuration with halved front trail $a=0.02 \mathbf{m}$; c) a configuration with doubled front trail $a=0.08 \mathbf{m}$.

The results are shown in Fig. 11-13 and are related to the real part of the eigenvalues; the imaginary part is not shown here since it does not add substantial information to the analysis.

Figure 11 shows the effect of front trail variation on the non-oscillating modes, i.e. the capsize and the rear wobble. It is worth noting that the capsize 


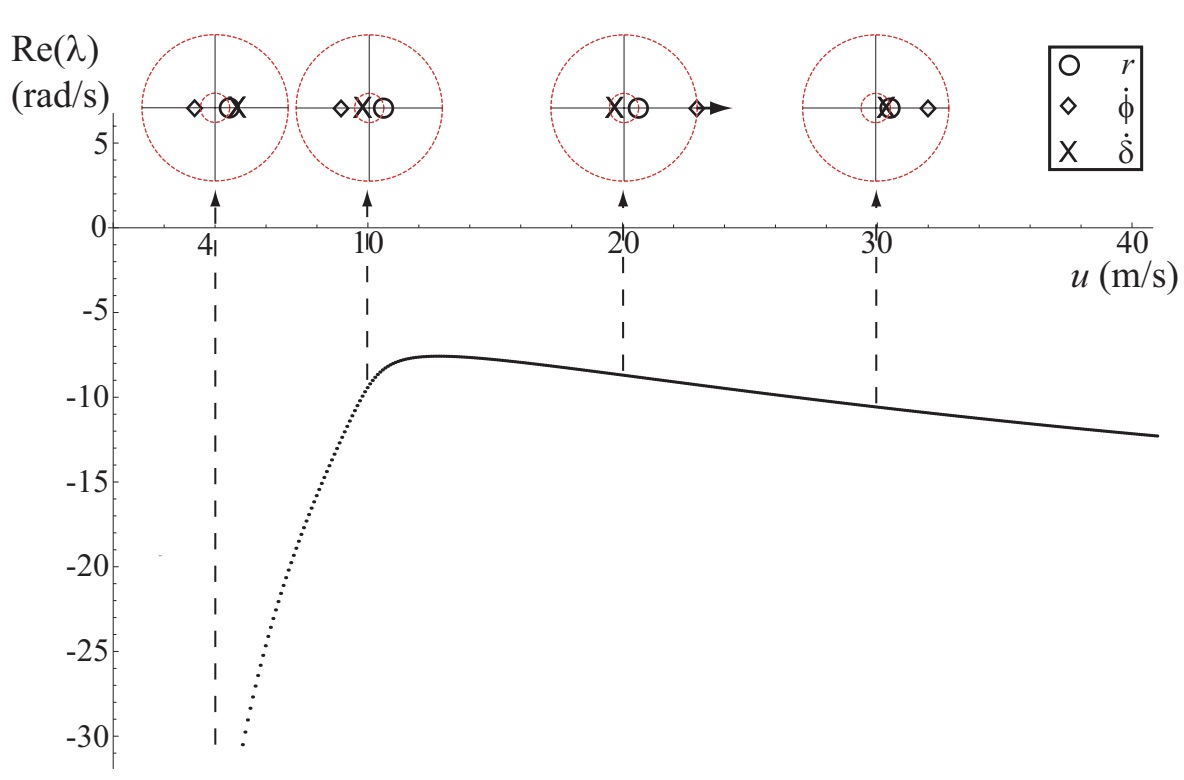

Fig. 10: Rear wobble eigenvalue and eigenvector representation versus longitudinal speed.

eigenvalue is always positive and it is not considerably affected by the front trail variation. On the contrary, the second wobble is strongly affected by the front trail value and it becomes more stable as the front trail value is reduced. It is worth noting that configuration c) shows a different behavior with respect to the other; indeed at longitudinal speed below $5 \mathrm{~m} / \mathrm{s}$ the rear wobble eigenvalue does not fall at very low (negative) value but it tends to zero as the longitudinal speed diminishes.

The weave versus longitudinal speed trend is shown in Fig. 12. Configurations a) and b) are very similar, it can be observed that the transition between non-oscillatory to oscillatory mode happens at $3-4 \mathrm{~m} / \mathrm{s}$ and the oscillatory weave mode related to the reference configuration is more damped at low speed (below $9 \mathrm{~m} / \mathrm{s}$ ) while it become less damped for higher speed values. Configuration c) shows a different trend: the transition between nonoscillatory and oscillatory mode happens at about $9 \mathrm{~m} / \mathrm{s}$ and the oscillatory mode is the most damped up to about $25 \mathrm{~m} / \mathrm{s}$.

Finally, the influence of the front trail on the wobble eigenvalue is shown in Fig. 13. The curves show that a different value of the front trail does not cause significant differences on wobble stability at low speed, while it significantly affects the speed at which the real part of the eigenvalue becomes positive. As it was already found for two wheelers, the greater the front trail the more stable the wobble mode. 


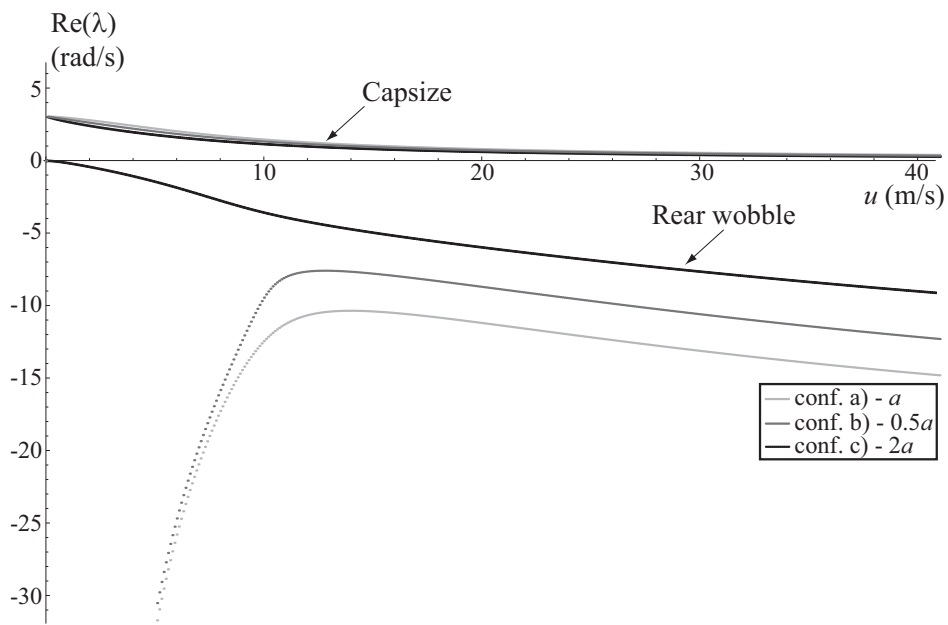

Fig. 11: Capsize and rear wobble modes - Front trail sensitivity analysis.

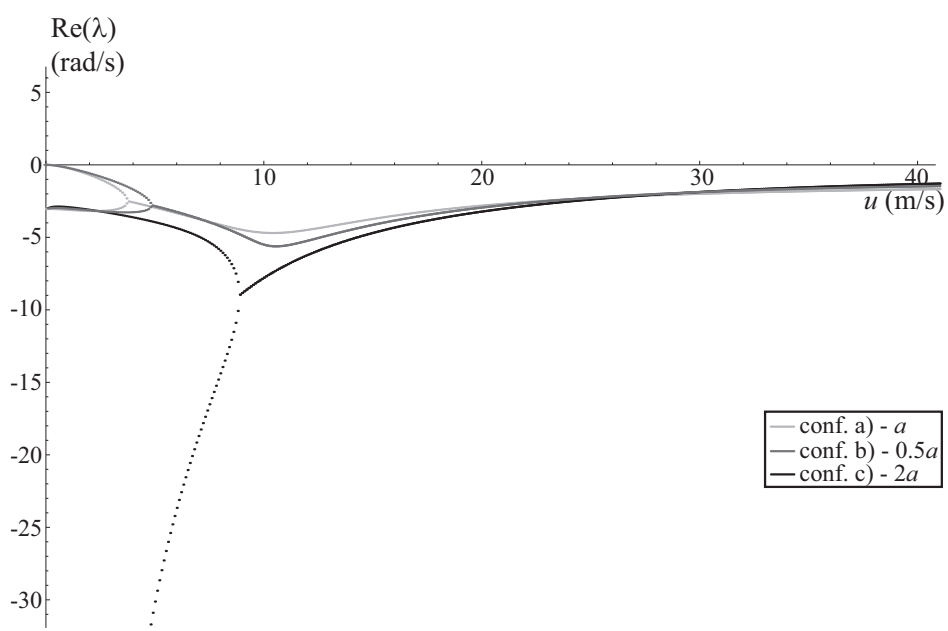

Fig. 12: Weave mode - Front trail sensitivity analysis.

\section{Conclusions}

In the present work the stability of a novel four wheeled tilting motorcycle was presented based on a mathematical model which did not consider the suspensions deflections and the tires radial stiffness. The stability analysis was discussed with reference to eigenvalues and eigenvectors in straight running. The developed model was validated by an equivalent simplified twowheeler version of the model itself, whose results were compared to those of a benchmark model which was found in the technical literature. 


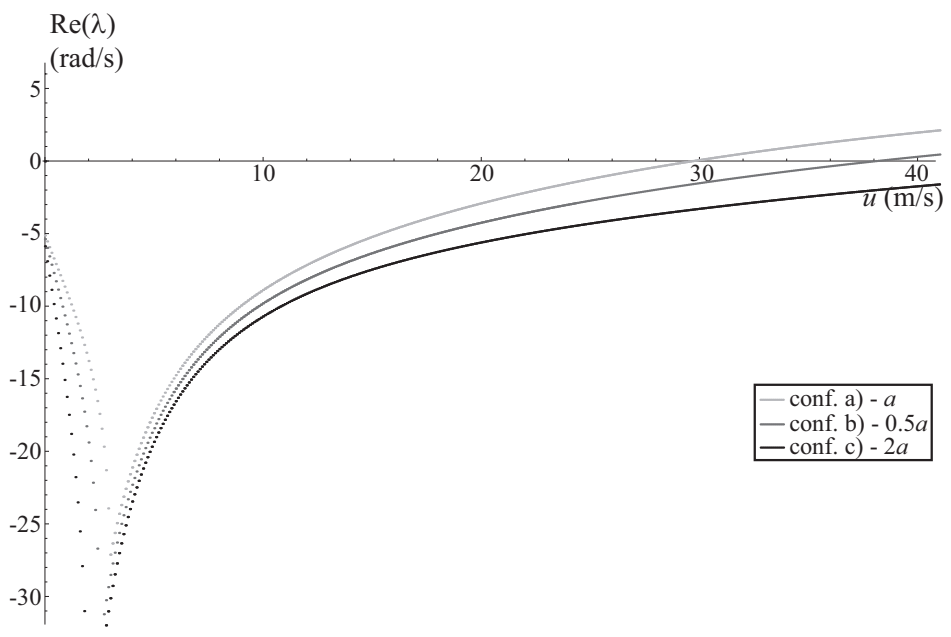

Fig. 13: Wobble mode - Front trail sensitivity analysis.

The eigenmodes which were found for the tilting four-wheeler were the well-known capsize, weave, wobble and rear wobble which characterize also two and three-wheelers. For the analysed vehicle the weave and wobble eigenmodes resulted stable and the capsize mode resulted unstable in the speed range from zero up to $38 \mathrm{~m} / \mathrm{s}$.

A smart graphical representation of the eigenvectors was also introduced to summarize in a single plot both the eigenvalue and the eigenvector information.

A sensitivity analysis to the front trail was also performed, showing the changes of the trend of the real part of the eigenvalues in straight running and the correlations between weave and rear wobble mode. It was shown that the front trail significantly affects almost all the natural modes; in particular, the weave and second wobble modes are influenced expecially at low speed, while the wobble mode shows a significantly different trend in the whole speed range, changing also the spied at which the eigenvalue becomes positive.

The modes which involve the suspension travel can not be analyzed by this simplified model and will be analyzed in the near future.

\section{Acknowledgments}

This works is inserts in the European Unions Horizon 2020 research and innovation programme under grant agreement No 653511.

\section{Compliance with Ethical Standards}

The project leading to this application has received funding from the European Unions Horizon 2020 research and innovation programme under grant 
agreement No 653511. The authors declare that they have no conflict of interest.

\section{References}

Sharp(1971). Sharp R S (1971) The stability and control of motorcycles, Journal of mechanical engineering science, 13(5), p. 316-329.

Sharp et al.(2004). Sharp R S and Evangelou S and Limebeer D J N (2004) Advances in the modelling of motorcycle dynamics, Multibody system dynamics, 12(3), p. 251-283.

Sharp and Limebeer(2001). Sharp R S and Limebeer D J N (2001) A motorcycle model for stability and control analysis, Multibody system dynamics, 6(2), p. 123-142.

Sharp(2001). Sharp R S(2001) Stability, control and steering responses of motorcycles, Vehicle system dynamics, 35(4-5) p. 291-318.

Frendo(2006). Frendo F and Sisi A and Guiggiani M and Di Piazza S (2006) Analysis of motorcycle models for the evaluation of the handling performances, Vehicle System Dynamics, 44(sup1), p. 181-191.

Nishimi et al.(1985). Nishimi T and Aoki A and Katayama T (1985) Analysis of straight running stability of motorcycles, SAE Technical Paper, 856124.

Limebeer et al.(2001). Limebeer D J N and Sharp R S and Evangelou S (2001) The stability of motorcycles under acceleration and braking, Proceedings of the Institution of Mechanical Engineers, Part C: Journal of Mechanical Engineering Science, 215(9), p. 1095-1109.

Cossalter and Lot(2002). Cossalter V and Lot R (2002) A motorcycle multi-body model for real time simulations based on the natural coordinates approach, Vehicle System Dynamics, 37(6), p. 423-447.

Cossalter et al.(2004). Cossalter V and Lot R and Maggio F (2004) The modal analysis of a motorcycle in straight running and on a curve, Meccanica, 39(1), p. 1-16.

Evangelou et al. (2008). Evangelou S and Limebeer D J and Rodriguez M T (2008) Influence of road camber on motorcycle stability, Journal of Applied Mechanics, 75(6), p. 061020.

Meijaard et al.(2007). Meijaard J P and Papadopoulos J M and Ruina A and Schwab A L (2007) Linearized dynamics equations for the balance and steer of a bicycle: a benchmark and review, Proceedings of the Royal Society of London A: Mathematical, Physical and Engineering Sciences, 463(2084), p. 1955-1982.

Sponziello et al.(2009). Sponziello A and Frendo F and Guiggiani M (2009) Stability analysis of a three-wheeled motorcycle, SAE International Journal of Engines, 1(1), p. 13961401 .

Bartaloni et al.(2007). Bartaloni F and Di Tanna O and Frendo F and Guiggiani M and Parenti M and Sponziello A (2007) Dynamic analysis of a novel three wheeled tilting vehicle, poster presentation at IAVSD 20th Symposium, Berkeley CA.

Rajamani and Gohl(2003). Rajamani R and Gohl J and Alexander L and Starr P (2003) Dynamics of narrow tilting vehicles, Mathematical and Computer modelling of dynamical systems, 9(2), p. 209-231.

Amati et al.(2011). Amati N and Festini A and Pelizza L and Tonoli A (2011) Dynamic modelling and experimental validation of three wheeled tilting vehicles, Vehicle System Dynamics, 49(6), p. 889-914.

Sponziello et al.(2010). Sponziello A and Frendo F and Guiggiani M and Rossi R (2010) Mass Damper Application to the Front Suspension of a Tilting Three Wheeler, SAE Technical Paper, no. 2010-32-0108.

EU Regulation(2013). (2013) Regulation (EU) No 168/2013 Annex I of the European Parlament and of the council of 15 January 2013, SAE Technical Paper, no. 2010-32-0108.

Cossalter et al.(2011). Cossalter V and Doria A and Lot R and Massaro M (2011) The effect of riders passive steering impedance on motorcycle stability: identification and analysis, Meccanica, 46(2), p. 279-292.

Guiggiani (2014). Guiggiani M (2014) The Science of Vehicle Dynamics: Handling, Braking, and Ride of Road and Race Cars, Springer Science \& Business Media. 